\title{
A Study on Applicability of Sound Art as Therapy for Alzheimer's Patients
}

\author{
Kamal Sabran ${ }^{1}$, Norfadilah Kamaruddin ${ }^{2}$, Ismail Lasa ${ }^{3}$, Norfarizah Mohd Bakhir ${ }^{1}$ \\ ${ }^{1}$ Universiti Sains Malaysia (USM) \\ ${ }^{2}$ Universiti Teknologi MARA (UiTM) \\ ${ }^{3}$ Ministry of Health Malaysia (MOH) \\ kamalsabran@gmail.com,norfadilah@salam.uitm.edu.my, drismaillasa@moh.gov.my,nmbakhir@gmail.com
}

\begin{abstract}
As Alzheimer's disease increases around the world with still no absolute medication to solve the problem, art has become the alternative treatment. Nevertheless, with sound art exploring public spaces to exhibit, and seen as not too distant from conventional art approach such as music therapy, the use of sound art is still not fully utilized to support a healthy environment in Alzheimer's care centres. This study aims to explore the applicability of sound art, a new medium of therapy for Alzheimer's patients. This project has investigated the issues, nature, and reasons that arise through literature reviews, pilot studies, observations and conducted interviews. In particular, it has focused on identifying the potential of sound characteristics, components and elements of sound art making. Views and opinions obtained from this method were used as data to form a prototype. This model has been tested on Alzheimer's patients as respondents in order to observe their perceptions, usability and affectivity. Finally, the data that gathered was analysed. Results from this research benefits the field of sound art in terms of new knowledge. The outcome of the project includes the identification of therapeutic ele-ments for sound art making and the empirical evaluation of its applicability use for Alzheimer's patients.
\end{abstract}

Keywords Therapy, Sound Art, Alzheimer's disease, New Media Arts, Interdisciplinary, Health

\section{Introduction}

In recent decades, arts have been connected to various fields of research such as science-technology, which includes healthcare research. Researchers have found evidence of the benefits of arts in healthcare in hospitals, nursing homes, senior centres, private homes, or other locations within the community. Arts in healthcare as a wide-ranging international movement that covers the waterfront of possibilities for how the arts enhance lives and impact patient care, hospital environments, care for caregivers, and community building within medical and other settings. [1]

Among others, the use of art as part of the therapeutic process for person with Alzheimer's disease is currently the most popular in this research field. Alzheimer's disease is a progressive, degenerative disease of the brain. There is no known cure and very few effective treatments for the disease. [2]

Various techniques of art therapy has been use such as painting, colouring, music listening and music therapy which according to researchers has positive feedback from a person with Alzheimer's disease as it will reduce isolation or withdrawal behaviour and provide an engaging experience to improve socialization, reduce restlessness or wandering, promote appropriate behaviour, improve word recall, stimulate long term memory skills, improve motivation, increase self-esteem, and provide an uplifting enjoyable feeling. [3]

The main objective of this research project is to investigate the validity of sound art as a new medium for art therapy for Alzheimer's patients. These outcomes will then provide a baseline for sound artist in their processes of producing or composing sound art for the Alzheimer's patients. Together, issue related to sound art and its benefits in Alzheimer's

disease therapy is then identified in order to obtain deeper understanding of this field.

\section{Problem Statement}

Previous research on art therapy for Alzheimer's disease shows several positive impacts but still has its limitation such as universality selection of the subject or theme and only temporarily effective for certain period of time. Other art related activities in Alzheimer's therapy program is not designed to be interactive as it is only one way or passive in nature. Thus, it needs a new approach, methods and techniques that specially design for the purpose of the Alzheimer's patients.

The previous study and research only focus on the visual art, music and other conventional artistic approach; therefore this research explored a new method using sound art and its possibilities. Sound art making involves interdisciplinary research characteristic explores the 
meaning of sound in everyday life. Thus, by identifying the right sound source, the best of sound composition and method of production; it has the potential to be explored for the benefit of Alzheimer's patients.

\section{Literature Reviews}

\subsection{Sound Art in Public Space}

Sound art in public space basically involves installing a space in another existing space, both physically and sensorial, and metaphysically and mentally - an interior space in an exterior space. The original quality of sound art lies in the oscillation of interior and exterior space. Thus public spaces intensified by sound art cause transitional spaces to come into being, in a political and a psychoanalytic sense. [4] Sound art expand it possibilities to be experienced by the audience with engagement in urban, public and networked space. In this sense, sound art can evoke a sense of being and place, which is strongly related to human visual experience. The auditory system has evolved to seek the reasons for the sound field it encounters as acoustic condition stimulates and enhances this response. [5]

\subsection{Sound and its Benefit to Human Health}

The use of sound and its relation to health have been at the centre of human interest from the beginning of recorded history. [6] The idea that creative expression can make a powerful contribution to the healing process has been embraced in many different cultures. However, the arts, specifically music, are normally identified as serving an aesthetic function, principally that of entertainment but their function as a therapeutic tool, however, is often neglected or, at the very best, misunderstood. [7]

\subsection{Art in Health Care Environment}

British Medical Association published a paper on "The psychological and social needs of patients" which found that creating a therapeutic healthcare environment extends beyond the elimination of boredom. [8] Arts and humanities programs have been shown to have a positive effect on patients. A study on the impact of art, design and environment in mental healthcare suggested that art can affect health, including physiological, psychological, clinical and behavioural effects. [9] Furthermore, art does not just give impact by encouraging beneficial clinical outcomes in patients, but it also enhances the quality of healthcare services and improves working conditions and job satisfaction. [10]

\subsection{Current Research on Art Therapy for People with Alzheimer's Disease}

Many researches indicated that auditory stimulation for people with Alzheimer's is effective for mood enhancement, relaxation, and cognition. Providing appropriate sensory stimulation for Alzheimer's disease has been shown in recent studies to decrease agitation and restlessness, as well as improve sleep. These symptoms are very common in most forms of dementia, and certainly in people with Alzheimer's, so sensory stimulation translates as improved quality of life for the patient as well as for the caregiver. [11]

\section{Methodology}

The main purpose of this research is to describe, explain and validate the sound art and its potential on Alzheimer's patients. Thus, description emerges following creative exploration, and serves to organize the findings in order to fit them with explanations, and then test or validate those explanations. Many research studies on Alzheimer's disease and its relationship to art have been done. Several important scientific discoveries as well as new information about the effectiveness of art as part of therapy program for Alzheimer's patients have resulted from making such descriptions. This process has then led to the discovery of new knowledge about value systems or practices of sound art as new medium in the fields.

To increase the essential understandings, this research project has engaged and employed qualitative approaches that can be related to case study research methodology. Following that, the researcher entered the field to examine and document what was occurring in the real situation. This is where ethnographic techniques were employed to gather empirical data, including face-to-face interview with doctors and caretakers, non-participant observation with the Alzheimer patients in care centre followed by focus group discussions. Finally, the researcher conducted a comprehensive analysis on the different data gathered to providenswers to the research questions that have been outlined.

\subsection{Research Design}

The research study was separated into three phases. The phases include the process of reviewing the established literature (Phase 1), data gathering process by conducting content analyses and field visits (Phase 2) and transforming all the information gathered into new findings by report writing (Phase 3). From this, two major outcomes could be established: a new set of sound art principles, characteristic and components that can serve to increase and effective for future research.

\subsection{Research Setting, Population and Sampling}

30 Alzheimer's patients from 2 institutional cares were observed and interviewed. These includes 6 caretakers and 4 doctors as respondent. The process involved observation and audio-video recording. Interviews were transcribed and analysed to obtain data. 


\subsection{Methods of Data Collection}

Through observation, numbers of sound art listening sessions for the Alzheimer's patients has been conducted in their natural setting. Alzheimer's care centres have provided the physical setting for non-participant care centre observations. They were limited to short-term commitments of three weeks for each of the two care centres. To ensure that patients did not feel distracted or uncomfortable during the observations, the researchers watched and took notes from a distance without interacting or participating. The resulting data was analysed inductively by interpreting the meaning of participants' perceptions as they occurred.

\section{Instrument Design}

The prototype of sound art is not just a work of art but it is also exploring the potentials of sounds and it frequency resonance to the patients.

\subsection{Soundscape for Alzheimer's Patients}

Based on the existing theory "The Preserved and Remaining Abilities in person with Alzheimer's Disease" [12] a sound art prototype developed based on a guideline according to their preserved emotional and musical capacities.

\subsection{Sound Art Design, Elements and Composition}

By applying theory of psychoacoustic, sound perception and sound therapy on to the sound art composition, the researcher believe the sound art could have the potential to be explored from respondent's views. There are 3 type of sound art prototype namely Nature Soundscape, Acoustic Instruments Soundscape and Abstract Soundscape specially design for the study.

\subsection{Sound Art Design, Elements and Composition}

Technique and methods are based on established sound art recording technique and methods developed by sound artist such as John Cage, Pierre Schaeffer, Terry Riley and La Monte Young. In this study, the researcher adopted methods from experimental music, Musique Concrete, and Electro-acoustic technique on to the sound art design prototype. The sound art composition also utilizes acousmatic sound as a compositional resource as suggested in Musique Concrete. There are two main factors in exploring sound as art namely extended technique where the techniques that are unconventional. Secondly the use of graphic notation; music composition written in the form of diagrams or drawings. [13]
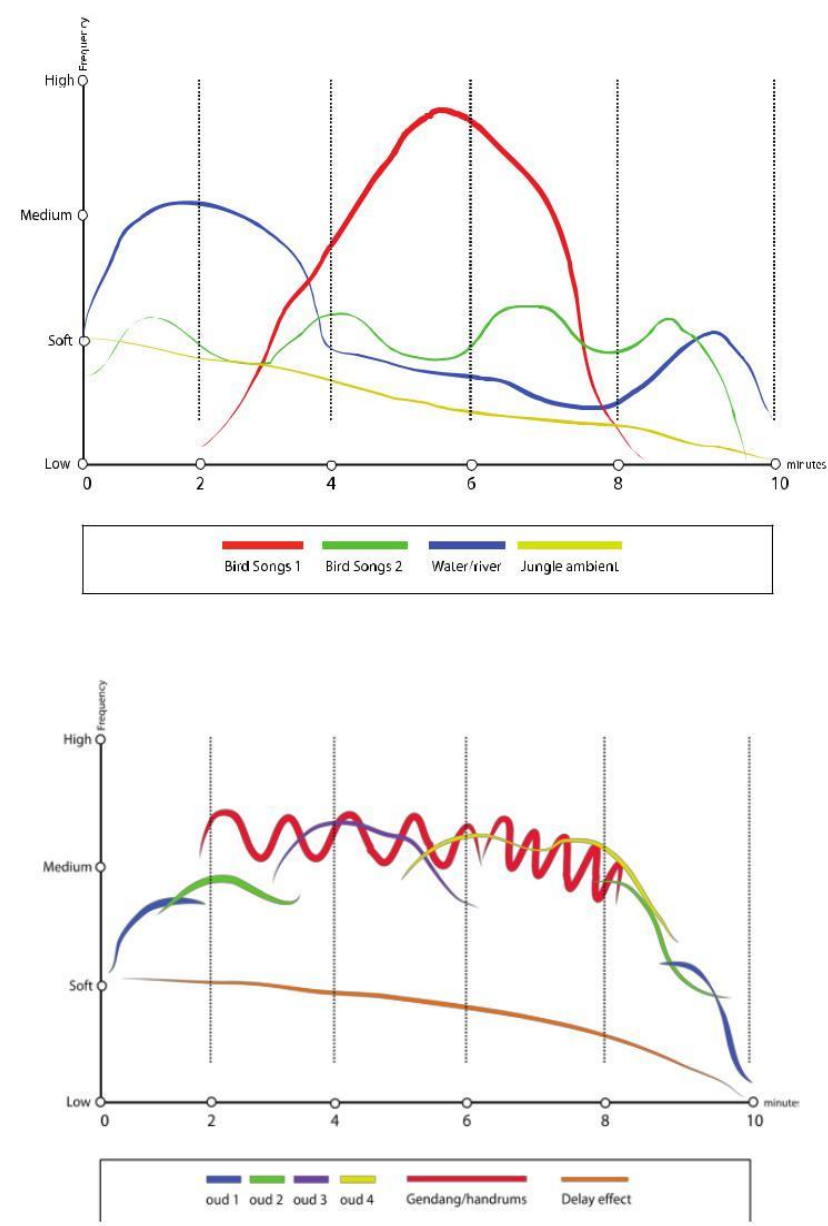

Figure 1. Graphic Notation for Sound Art Composition

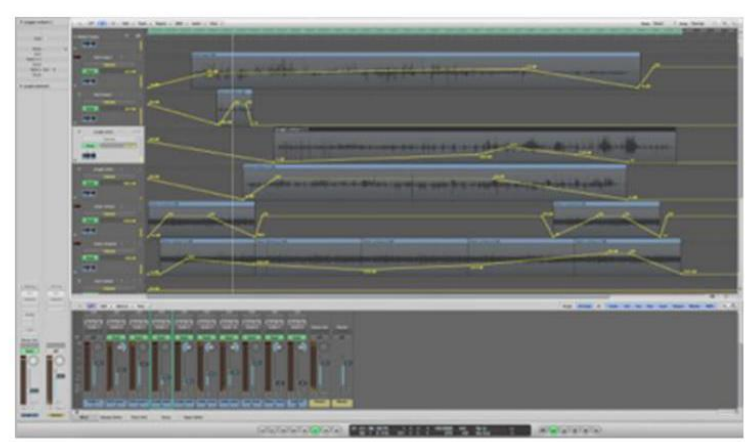

Figure 2. Sound Art Composition

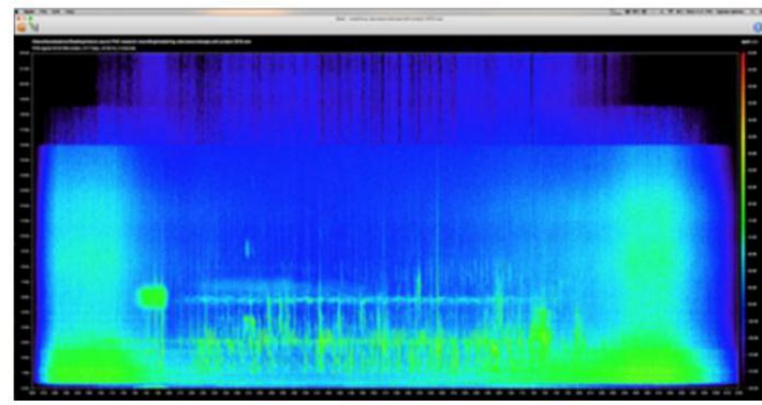

Figure 3. Sound Art Audio Spectogram Analysis 


\section{Data Collection}

The fieldwork involved processes supported with ethnographic methods, undertaken in Alzheimer's care centres.

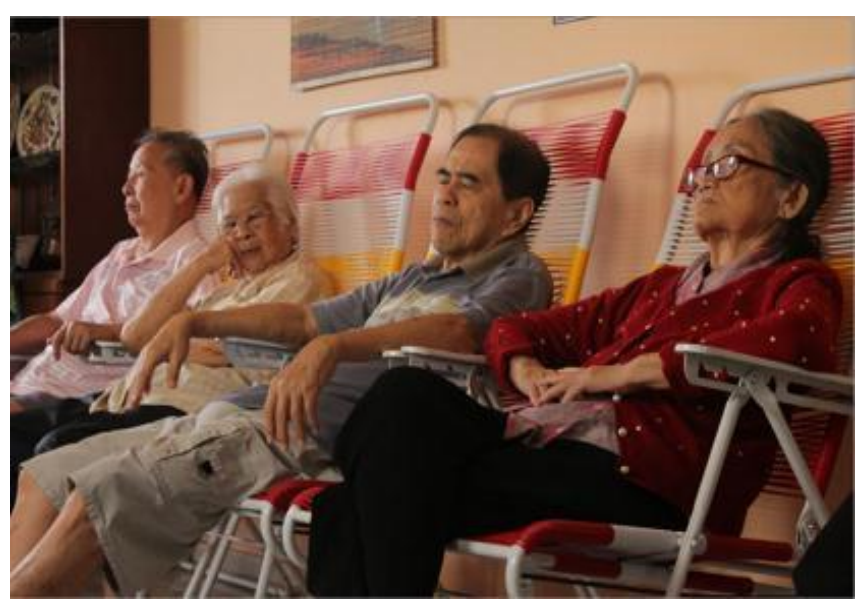

Figure 4. Alzheimer's patients listening to sound art in their room.

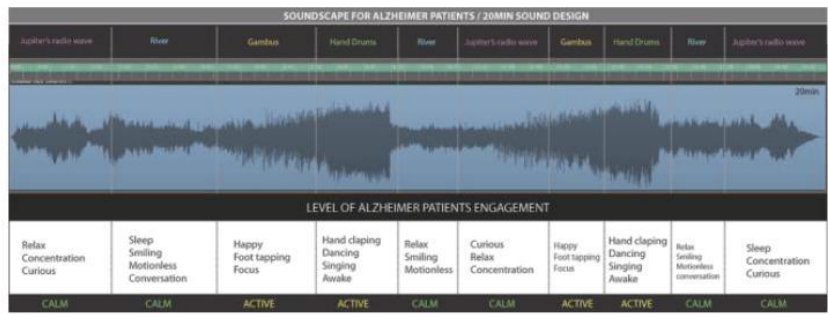

Figure 5. Patients responses and level of engagement in sound art components.

In essence, the data is presented in relation to three types of sound art design which makes the Alzheimer's Soundscape. With regards to the level of Alzheimer's patients responds to the sound art given, various patients reaction was recorded during the 20 minutes listening session with total 3 times per day for 4 weeks' duration and this can be traced based on physical responses, verbal responses, mood and other responses.

\section{Findings}

The observations found significant sign of engagement from patients towards sound art listening session and the patterns of behaviour below:

\subsection{Sound Art Type 1: Nature Soundscape}

During the observations, patients frequently looks motionless but in a relax position. It seemed that patients were much more responsive to the sound of nature by judging to the physical and verbal responses. It was also evident that all patients appeared to be active, comfortable and without facial grimes and tension. Although there were a few patients (stage four Alzheimer's Disease) is less responsive through physical or verbally.

\begin{tabular}{|l|l|l|l|}
\hline $\begin{array}{l}\text { Sound Art } \\
\text { Type }\end{array}$ & Physical & Verbal Responses & Mood \\
\hline Nature sound- & Relax & Communicating & Comfort \\
scape & Smiling & Asking & Curiosity \\
& Focus & Questions & Attention \\
& Closed eyes & Telling stories & \\
\hline
\end{tabular}

Table 1. Level of patients' responses through physical, verbal and mood while listening to the Alzheimer's Soundscape: Nature Soundscape.

\subsection{Sound Art Type 2: Acoustic Soundscape}

From the observation, most of them are physically active in reaction to the sound art. It was also evident that all patients appeared to be comfortable and without facial grimes and tension. The researcher noticed that the sound of hand drums that created consistence beat make them enjoy it more. They were having less interaction to each other as they are more focused on the sound. During the observations, patients commonly looks happy, interested and engaged.

\begin{tabular}{|l|l|l|l|}
\hline $\begin{array}{l}\text { Sound Art } \\
\text { Type }\end{array}$ & $\begin{array}{l}\text { Physical } \\
\text { Responses }\end{array}$ & Verbal Responses & Mood \\
\hline $\begin{array}{l}\text { Acoustic } \\
\text { soundscape }\end{array}$ & Toe tapping & Less talking & Active \\
& Hand clapping & & Happy \\
& Dancing & & Interest \\
& Head shaking & & Engaged \\
& & & \\
\hline
\end{tabular}
while listening to the Alzheimer's Soundscape: Acoustic Soundscape.

\subsection{Sound Art Type 3: Abstract Soundscape}

The observations found sign of disengagement of the pa-tients towards soundscape listening session. Mostly patients were very passive and motionless throughout the listening session. From the observation, most of them are physically inactive. The reaction was contrasting with the other two types of soundscape given. However, there are one interesting finding during the observations, patients commonly look very calm and sleepy.

\begin{tabular}{|l|l|l|l|}
\hline $\begin{array}{l}\text { Sound Art } \\
\text { Type }\end{array}$ & $\begin{array}{l}\text { Physical } \\
\text { Responses }\end{array}$ & Verbal Responses & Mood \\
\hline $\begin{array}{l}\text { Abstract } \\
\text { soundscape }\end{array}$ & $\begin{array}{l}\text { Motionless } \\
\text { Sleepy }\end{array}$ & $\begin{array}{l}\text { Silence } \\
\text { Complaining }\end{array}$ & $\begin{array}{l}\text { Passive } \\
\text { Bored }\end{array}$ \\
& Calm & & \\
\hline
\end{tabular}

Table 3. Level of patients' responses through physical, verbal and mood while listening to the Alzheimer's Soundscape: Abstract Soundscape.

Based on the observations that these 3 types of sound art components were by far the most likely to trigger responses, such as body movement, facial expression and verbally such as asking questions or start to have conversation to each other. This phenomenon suggested that the patients were trying to interpret the meaning of sound that they engaged with in the care centre environment. 


\begin{tabular}{l|c|c|c|c|c|c|c|c|c}
\hline \multicolumn{8}{c|}{ Sound component type and engagement } \\
$\begin{array}{l}3 \text { stage } \\
\text { Alzheimer's } \\
\text { disease }\end{array}$ & \multicolumn{3}{c|}{ Nature } & \multicolumn{3}{c}{ Acoustic instruments } & \multicolumn{3}{|c}{ Abstract } \\
\hline Mild & & & $*$ & & & $*$ & $*$ & & \\
\hline Moderate & & $*$ & & & $*$ & & $*$ & & \\
\hline Severe & $*$ & & & $*$ & & & $*$ & &
\end{tabular}

Table 4. Patients engagement with various sound art component

\section{Conclusion}

Sound art is an interdisciplinary art form that has great potential to be explored in public spaces especially in health care environments. With the advance of technology, sound art could be part of the sonic environment in care centre, which would benefit the patients, medical staff and so on by creating a healthy environmental soundscape. The research finding shows that Alzheimer's patients still could respond to sound art despite of memory lost due the disease. Instead, most of the doctors, and caregivers agreed that sound art is beneficial and could be permanently installed in Alzheimer's care centre for positive impact. Based on the research data, it shows that most of the patients positively react to the sound art given. From the interviews with doctors and caregivers mentions that sound art has similar effect to music therapy. Thus, the results have shown that sound art have therapeutic effects and can be elicited through artistic involvement in Alzheimer's patients.

Data from the study also indicates that it is not just patients who react positively toward the sound art, but it also helps in creating vibrant health care environments that benefit the people surround such as medical staff, and caretakers. Several doctors and medical staff stated that sound art have a great potential in achieving effective approaches to patient management and to the education and training of health practitioners. It identifies the relative contribution of dif-ferent art forms to the final aim of creating a therapeutic healthcare environment.

As an addition to the field of interdisciplinary arts, this research looking in depth into sound art and its applicability as therapy for Alzheimer's patients in the care centre environments by focusing on characteristic and the new form of sound art making. This study is based on research done on new media arts in the context of today's technology. It would be very interesting if in the future, the research will involve in depth study of characteristics or theories evolve in time.

\section{REFERENCES}

[1] Cathy, M. (2010). Arts in Healthcare: Creativity for the Health of It. Psychology Today. Retrieved on 22 January 2016. 5:30AM from http://psychologytoday.com
[2] Judith, N., Sophia, W. (2012). Alzheimer's Disease: Diagnosis and Treatment Across the Spectrum of Disease Severity. Retrieved on November 8, 2015. 2am from http://www.ncbi.nlm.nih.gov

[3] Amy, N. (2013). Music as medicine. American Psychological Association. Retrieved on 20 February 201. 5:20AM from http://www.apa.org

[4] George, K. (2009). Site-sounds: on strategies of sound art in public space. Retrieved on February 7, 2016. 2:01AM from www.georgklein.de

[5] Simon, E. (2007). Living electronic music. New York: Routledge.

[6] Heather L. S and Jeremy, N. (2010). The Connection Between Art, Healing, and Public Health: A Review of Current Literature. Retrieved on October 2015. 1:30AM from www.ncbi.nlm.gov

[7] Mohamed Ghouse Nasuruddin (2010). The confluence between arts and medical science: music and movement therapy for children with cerebral palsy. Retrieved on Jan 16, 2013, 2:50AM from www.ncbi.nlm.nih.gov

[8] British Medical Association (2011). The psychological and social needs of patients. Retrieved on 25 May 2010. 2:30AM from www.ahsw.org.uk

[9] Norma, D., Ellie B., Tony, S., Susan, O. (2008). Review: the impact of art, design and environment in mental healthcare: a systematic review of the literature. Retrieved on November 7, 2014. 4:12AM from http://rsh.sagepub.com

[10] Rosalia, L. S. (2004). Arts in health: a review of the medical literature. Retrieved on February 7, 2016. 2:01AM from www.creativenz.govt.nz

[11] Johnson, C., Lahey, P., Shore, A. (1992). An exploration of creative arts therapeutic group work on an Alzheimer's unit. Arts in Psychotherapy;19, 4, 269-277.

[12] Eilon, C. (2014 Remaining Abilities in Persons with Alzheimer's Disease. Retrieved on Jan 4, 2014 from http://dementiabehaviorconsulting.com

[13] John Cage. (1973). Silence. USA: Wesleyan University Press. 